\title{
Is lumbar facet fusion biomechanically equivalent to lumbar posterolateral onlay fusion?
}

\author{
Jeffrey M. Toth, PhD, ${ }^{1}$ Kevin T. Foley, MD, ${ }^{2}$ Mei Wang, PhD, ${ }^{1}$ Howard B. Seim III, DVM, ${ }^{3}$ and \\ A. Simon Turner, BVSc, MS ${ }^{3}$

\begin{abstract}
'Department of Orthopaedic Surgery, The Medical College of Wisconsin Inc., Milwaukee, Wisconsin; ${ }^{2}$ Department of Neurosurgery, University of Tennessee Health Science Center, Memphis, Tennessee; ${ }^{2}$ Department of Clinical Sciences, Colorado State University, Fort Collins, Colorado
\end{abstract}

\begin{abstract}
OBJECTIVE This study was designed with the following research objectives: 1) to determine the efficacy of facet fusion with recombinant human bone morphogenetic protein-2 (rhBMP-2) on an absorbable collagen sponge (ACS) in an ovine lumbar facet fusion model; 2 ) to radiographically and histologically compare the efficacy of lumbar facet fusion with rhBMP-2/ACS to facet fusion with an iliac crest bone graft (ICBG); and 3) to biomechanically compare lumbar facet fusion with rhBMP-2/ACS to lumbar posterolateral fusion (PLF) with ICBG.
\end{abstract}

METHODS The efficacies of the 3 treatments to induce fusion were evaluated in an instrumented ovine lumbar fusion model. Eight sheep had $10 \mathrm{~cm}^{3} / \mathrm{side}$ ICBG placed as an onlay graft for PLF at L2-3. At the adjacent L3-4 level, $0.5 \mathrm{~cm}^{3} /$ side ICBG was placed for facet fusion. Finally, $0.5 \mathrm{~cm}^{3} / \mathrm{side}$ rhBMP-2/ACS $(0.43 \mathrm{mg} / \mathrm{ml})$ was placed for facet fusion at L4-5. CT scans were obtained at 2, 4, and 6 months postoperatively with 2 reviewers conducting an evaluation of the 6 -month results for all treated spinal levels. All 8 sheep were killed at 6 months, and all posterolateral instrumentation was removed at this time. The spines were then sectioned through L3-4 to allow for nondestructive unconstrained biomechanical testing of the L2-3 and L4-5 segments. All treated spinal levels were analyzed using undecalcified histology with corresponding microradiography. Statistical comparisons were made between the treatment groups.

RESULTS The PLF with ICBG (ICBG PLF group) and the rhBMP-2 facet fusion (rhBMP-2 Facet group) treatment groups demonstrated similar levels of stiffness, with the rhBMP-2 Facet group having on average slightly higher stiffness in all 6 loading directions. All 8 levels in the autograft facet fusion treatment group demonstrated CT radiographic and histological fusion. All 8 levels in the rhBMP-2 Facet group showed bilateral CT radiographic and histological fusion. Six of 16 rhBMP-2/ACS-treated facet defects demonstrated small intraosseous hematomas or seromas. Four of the 8 levels $(50 \%)$ in the ICBG PLF treatment group demonstrated bilateral histological fusion. Three of 8 levels in the ICBG PLF treatment group showed unilateral fusion. One of the 8 levels in the ICBG PLF treatment group demonstrated bilateral histological nonfusion.

CONCLUSIONS Both rhBMP-2/ACS and autograft demonstrated 100\% efficacy when used for facet fusion in the instrumented ovine model. However, the ICBG PLF treatment group only demonstrated a 50\% bilateral fusion rate. Biomechanically, the ICBG PLF and rhBMP-2 Facet groups demonstrated similar stiffness in all 6 loading directions, with the rhBMP-2 Facet group having on average slightly higher stiffness in all directions.

https://thejns.org/doi/abs/10.3171/2016.10.SPINE16649

KEY WORDS facet fusion; posterolateral fusion; onlay grafting; recombinant human bone morphogenetic protein-2; spinal fusion; bone morphogenetic proteins; lumbar; degenerative; sheep

$\mathrm{I}$ $\mathrm{N}$ a retrospective analysis of uninstrumented facet fusions in patients undergoing lumbar laminectomy for spinal stenosis due to single-level degenerative spondylolisthesis, Pateder and Benzel found that uninstrumented facet fusion decreases motion and stabilizes the spine..$^{11} \mathrm{In}$ addition, clinically, a facet fusion is less invasive, requires less bone graft harvest, and is associated with less morbid- ity than posterolateral fusion (PLF) using autograft.,10,11 However, it is not known if the biomechanical stiffness of a facet fusion is equivalent to the biomechanical stiffness of a posterolateral onlay fusion. In addition, although osteoinductive bone graft substitutes avoid autograft harvest, it is not known if an osteoinductive bone graft substitute would be efficacious in inducing a facet fusion.

ABBREVIATIONS ACS = absorbable collagen sponge; ICBG = iliac crest bone graft; PLF = posterolateral fusion; rhBMP-2 = recombinant human bone morphogenetic protein-2.

SUBMITTED June 21, 2016. ACCEPTED October 11, 2016.

INCLUDE WHEN CITING Published online February 3, 2017; DOI: 10.3171/2016.10.SPINE16649. 


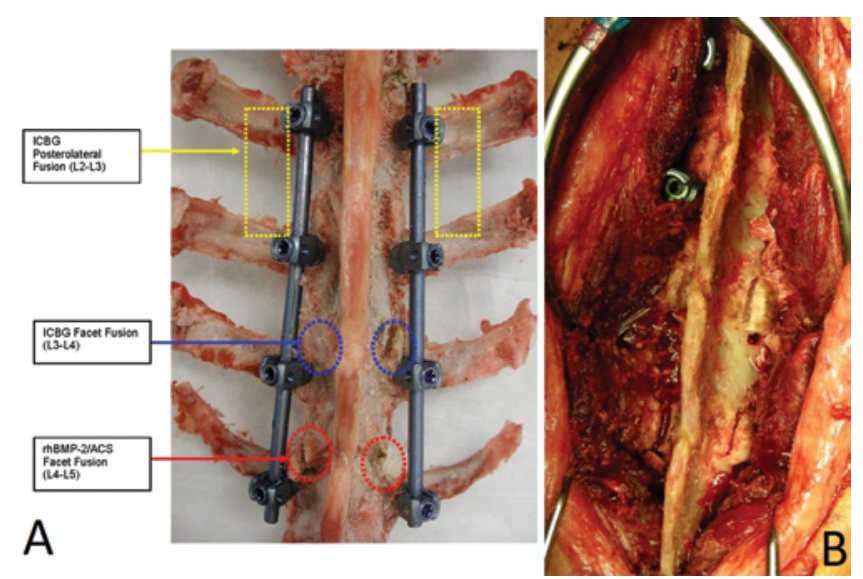

FIG. 1. A: Photographs showing the locations of the treatment, including the locations of the PLFs at L2 -3 , the bilateral facetectomies at L3-4 and $L 4-5$, and the supplemental spinal instrumentation used in this study. B: Intraoperative photograph showing the bilateral facetectomies at L3-4 and L4-5, as well as the medial placement of ICBG at L2-3. Figure is available in color online only.

Our study was designed with the following research objectives: 1) to determine the efficacy of facet fusion with recombinant human bone morphogenetic protein-2 (rhBMP-2) on an absorbable collagen sponge (ACS) in an ovine lumbar facet fusion model; 2) to radiographically and histologically compare the efficacy of lumbar facet fusion with rhBMP-2/ACS to lumbar facet fusion with iliac crest bone graft (ICBG); and 3) to biomechanically compare lumbar facet fusion with rhBMP-2/ACS to PLF with ICBG. CT radiography, nondestructive biomechanics, and undecalcified histology with corresponding microradiography were used to evaluate the efficacies of these treatments to induce fusion in the instrumented ovine lumbar spine model.

\section{Methods}

\section{Animal Model}

The ovine lumbar spine model was specifically chosen because of the biomechanical similarities between the ovine and human lumbar spine., ${ }^{2,12,13,18}$ Wilke et al. ${ }^{18}$ characterized the biomechanical parameters (range of motion, neutral zone, and stiffness parameters) of sheep spines and made comparisons with data from human specimens that were previously published by White and Panjabi. ${ }^{17}$ Wilke et al. found that the "ranges of motion of sheep spines for the different load directions are qualitatively similar in their craniocaudal trends to those of human specimens reported in the literature."18 They concluded that "based on the biomechanical similarities of the sheep and human spines demonstrated in this study, it appears that the sheep spine ... can serve as an alternative for the evaluation of spinal implants." ${ }^{18}$ We have shown that the ovine lumbar spine model has a biomechanical response that is similar to the human spine. ${ }^{2}$

\section{Materials and Study Design}

The animal model consisted of 8 skeletally mature female sheep. This study was approved by the Institutional Animal Care and Use Committee and was conducted under the recommendations of the American College of Laboratory Animal Medicine and the Public Health Service Guide for the Care and Use of Laboratory Animals. The sheep were fasted for 24 hours prior to surgery. Water was not restricted during this time. Anesthesia was induced with ketamine (4 mg/kg) and valium (7.5 mg total). After induction, sheep were maintained with isoflurane $(1.5 \%-3 \%)$ in $100 \%$ oxygen $(2 \mathrm{~L} / \mathrm{min})$ during the surgical procedure. Muscle relaxants were not used. The locations of the treatments can be seen in Fig. 1A.

With the animal in sternal recumbency and under general endotracheal anesthesia while using aseptic technique, a dorsal midline incision was made to expose the lumbodorsal fascia and palpable spinous processes from L-1 to L-6. The fascia was split by sharp dissection, and a dissection plane was further developed from the spinous processes to the transverse processes. The transverse processes of L-2 and L-3 and the pars interarticularis were cleared of soft tissues using electrocautery. As shown in Fig. 1B, the L2-3, L3-4, and L4-5 facet joints were excised bilaterally with the aid of a high-speed bur. Decortication of the dorsal aspect of the L-2 and L-3 lateral pars and transverse processes was performed using a high-speed bur.

All 8 sheep received identical treatments at adjacent levels from L2-L3 to L4-L5 as summarized in Table 1 and illustrated in Fig. 1A and 1B. At L2-3, all 8 sheep had $10 \mathrm{~cm}^{3} /$ side ICBG placed as an onlay graft bilaterally in the paraspinal bed from the region spanning the L2-3 transverse processes and along the lateral pars area. At L3-4, all 8 sheep had $0.5 \mathrm{~cm}^{3} /$ side ICBG placed for facet fusion after bilateral facetectomy. Finally, after bilateral facetectomy, all 8 sheep had $0.5 \mathrm{~cm}^{3} /$ side rhBMP-2 (0.43 $\mathrm{mg} / \mathrm{mL}$ ) on an ACS placed for facet fusion at L4-5. The release characteristics of rhBMP-2 from the ACS have been studied using ${ }^{125}$ I-radiolabeled rhBMP-2 in a rabbit ulna osteotomy model..$^{5}$ In that model, approximately $33 \%$ of ${ }^{125}$ I-radiolabeled rhBMP-2 was retained at the defect after 7 days, and less than $10 \%$ of ${ }^{125}$ I-radiolabeled rhBMP-2 was retained at the defect after 14 days. ${ }^{5}$ The fusion site was stabilized using bilateral transpedicular instrumentation (CD Horizon Legacy Spinal System; Medtronic Spinal and Biologics). The wounds were then closed in layers. Postoperative pain management and wound care was provided until the animals returned to activities ad libitum. Pain medication after the surgical procedures included fentanyl patches at a dose of $150 \mathrm{mg} / \mathrm{hr}$ administered with a continuous percutaneous patch for 3 days. Additional pain medication included phenylbutazone at a dose of $1 \mathrm{~g}$ administered orally once per day for 3 days. At the conclusion of the study, all of the sheep were killed by barbiturate overdose at 6 months postoperatively. At necropsy, the spine was harvested en bloc from L-1 to L-7. All instrumentation was then removed, and the spines were carefully sectioned through L3-4 (without damaging the bilateral facet fusion at that level) to allow for nondestructive unconstrained biomechanical testing of the L2-3 and L4-5 segments. All spinal levels were also analyzed using undecalcified histology with corresponding microradiography.

\section{Radiography and CT}

A high-resolution radiography unit (Faxitron mod- 
TABLE 1. Table of treatments

\begin{tabular}{clll}
\hline Treated Level & \multicolumn{1}{c}{ Treatment } & Abbreviation & \multicolumn{1}{c}{ Methods of Evaluation } \\
\hline L2-3 & $10 \mathrm{~cm}^{3} /$ side ICBG PLF & ICBG PLF & CT, nondestructive biomechanical testing, \& undecalcified histology \\
\hline L3-4 & $0.5 \mathrm{~cm}^{3} /$ side ICBG facet fusion & ICBG Facet & CT \& undecalcified histology \\
\hline L4-5 & $0.5 \mathrm{~cm}^{3} /$ side rhBMP-2/ACS facet fusion & rhBMP-2 Facet & CT, nondestructive biomechanical testing, \& undecalcified histology \\
\hline
\end{tabular}

el 43805; Hewlett Packard) and high-resolution film (EKTASCAN B/RA Film 4153; Kodak) were used to produce a high-resolution posteroanterior radiograph of the lumbar spine prior to biomechanical testing. CT imaging was completed using parameters appropriate for bone at a 1-mm slice thickness. Sagittal and coronal plane reconstructions were obtained for all treated sheep. CT scans were evaluated by 2 independent reviewers. Based on both the right and left sides of the coronal CT slices for the spinal level, the evaluators were asked to rate an overall fusion score for that level using the following criteria: a score of 3 indicated solid PLF on the right and left sides with no radiolucencies interrupting continuous superiorto-inferior bony bridging. A score of 2 indicated unilateral fusion with PLF on the right or left side, but not both. A score of 2 indicated that lucencies were present in the posterolateral space on the right or left side that interrupted continuous superior-to-inferior bony bridging. A score of 1 indicated nonfusion with isolated bone formation without continuous superior-to-inferior bony bridging on both the right and left sides. A score of 1 indicated bilateral lucencies with no evidence of continuous superior-to-inferior bony bridging (posterolateral fusion) on the right or left side. Facet fusion was defined as continuous bony bridging dorsal to the facet joint on CT.

\section{Nondestructive Biomechanical Testing}

After all instrumentation was removed, the 8 lumbar spines were carefully sectioned in the axial plane through L3-4 (without damaging the bilateral facet fusion at that level) to allow for nondestructive unconstrained biomechanical testing of the L2-3 and L4-5 spinal segments. No biomechanical testing was conducted on the L3-4 segments. Unconstrained biomechanical testing was performed in a nondestructive manner on all 8 L2-3 and L4-5 levels to determine the stiffness of the motion segments in flexion, extension, right and left lateral bending, and right and left axial rotation. Specially designed loading and base fixtures were secured to the treated levels. Moments of $0,0.5,2.5,4.5,6.5$, and $8.5 \mathrm{Nm}$ were achieved in each loading direction by applying static loads used to generate the pure moments. A 6 degree-of-freedom load cell was placed in series with the specimens to verify the applied moments. Three-dimensional rotation of the sheep lumbar spine motion segments was obtained using a motion analysis system (Optotrak Certus Motion Tracking System; Northern Digital Inc.). Three active markers were attached to each vertebra. The locations of these markers were recorded with the motion analysis system at each load. The 3D coordinate data were analyzed to obtain the rotation angles of the superior vertebra with respect to the inferior vertebra, generating the rotational flexibility (stiffness) of each motion segment. The stiffness (flexibil- ity) of each motion segment was derived using linear regression from loading levels of 0.5 to $6.5 \mathrm{Nm}$. In addition, historical biomechanical data from a normal intact group of sheep lumbar spine motion segments were used as baseline data for normal lumbar spine motion in sheep. ${ }^{2}$ Differences in the segmental stiffness between the 2 fusion treatments and the normal intact spines were statistically compared. Immediately following biomechanical testing, all spinal levels were fixed in $10 \%$ neutral buffered formalin in preparation for undecalcified histology as well as corresponding microradiography.

\section{Undecalcified Histology}

After biomechanical testing, the anterior column was removed and the dorsal tissues of the right and left intertransverse process spaces and medial spine (for L2-3) and the right and left facet joints (for L3-4 and L4-5) were labeled and processed for undecalcified histology. Tissues were dehydrated in graded alcohols, cleared in xylene, and infiltrated and embedded in graded catalyzed methyl methacrylate. After polymerization was complete and the samples had hardened, the blocks containing the fusion masses were continuously sectioned in the sagittal plane on diamond saws. Approximately 10-20 sections were made in the sagittal plane through each half of the treated level. Differential staining using a proprietary trichrome stain was performed to permit both histological and cytological differentiation. With this staining method, the following tissues can be differentiated based on color: bone is stained blue/green, cartilage and fibrocartilage are stained dark purple, and fibrovascular tissue is stained pink. Staining the cellular and nuclear detail with the trichrome stain is similar to $\mathrm{H} \& \mathrm{E}$, thus permitting cytological differentiation. Differential staining along with qualitative optical microscopy was performed to assess bony bridging and the extent of fusion associated with the autograft and the bone graft substitute. Histological examination was not only used to assess continuous bony bridging but also to evaluate the histological response to the treatments, including the presence or absence of an inflammatory response, the presence or absence of bone remodeling, and the fate of the bone graft/substitute. Corresponding microradiography was conducted on several sections from each treated level. These were analyzed concurrently with stained undecalcified sections for fusion determination. Only spinal levels that showed continuous bony bridging from superior to inferior were considered fused.

\section{Statistical Analysis}

The biomechanical stiffness data for each loading direction were statistically analyzed using ANOVA. A statistical significance level of $\mathrm{p}<0.05$ was used for ANO- 

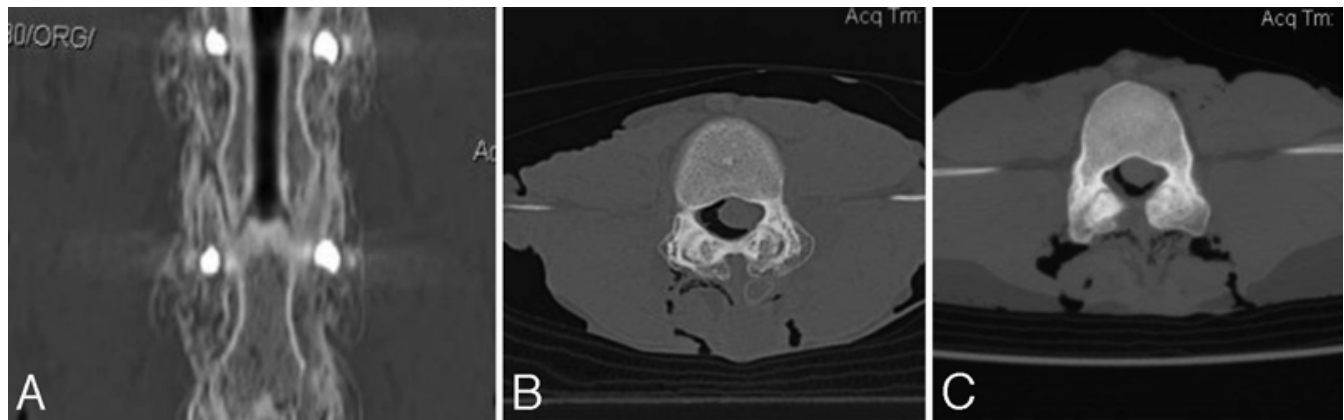

FIG. 2. A: Coronal CT scan obtained 6 at months, showing a bilateral PLF at the L2-3 spinal level treated with ICBG. B: Representative axial CT scan obtained at 6 months, showing a facet fusion treated with ICBG at L3-4. C: Representative axial CT scan obtained at 6 months, showing a facet fusion treated with rhBMP-2/ACS at L4-5.

VA. If ANOVA indicated that statistical significance was found, further pairwise comparisons of the biomechanical data were then made using the Bonferroni-Dunn post hoc test. A statistical significance level of $p<0.0167$ was used for further pairwise comparisons when using the Bonferroni-Dunn post hoc test.

\section{Results}

\section{Computed Tomography}

All facetectomy defects treated with ICBG at L3-4 and all facetectomy defects treated with rhBMP-2/ACS at L4-5 were fused with bridging trabecular bone across the facetectomy defects. Evaluator 1 found 3 solid fusions, 4 unilateral fusions, and 1 nonfusion in the ICBG PLF group. Evaluator 2 found 6 solid fusions and 2 unilateral fusions in the ICBG PLF group. PLF masses were found to be very medial. These fusion masses did not extend laterally into the middle of the intertransverse process space. A 6-month coronal CT slice showing bilateral posterolateral fusion at the L2-3 spinal level treated with ICBG is seen in Fig. 2A. Representative 6-month axial CT slices of facet fusions treated with ICBG at L3-4 and rhBMP-2/ ACS at L4-5 are seen in Fig. 2B and 2C, respectively.

\section{Biomechanical Testing}

The extension test results from 1 specimen in the ICBG PLF group was lost due to a technical error, so analysis of the extension load direction was performed with only 7 specimens $(n=7)$. The mean $(+S D)$ stiffness of the fusion segments for the 2 biomechanically tested treatment groups (rhBMP-2 Facet and ICBG PLF), as well as the normal intact spine segments (historical data), are presented in Fig. 3. The 2 treatment groups demonstrated similar stiffness in all 6 loading directions, with rhBMP2 Facet having on average slightly higher stiffness in all 6 directions. The only difference between the rhBMP-2 Facet and ICBG PLF groups that reached the level of statistical significance was the stiffness in extension $\left(9.6 \mathrm{Nm} /{ }^{\circ}\right.$ vs $7.5 \mathrm{Nm} /{ }^{\circ}$, respectively; $p<0.01$ according to the Bonferroni-Dunn post hoc test). Compared with the intact spines, both treated groups were significantly stiffer in all loading directions $(\mathrm{p}<0.0001$ according to the Bonferroni-

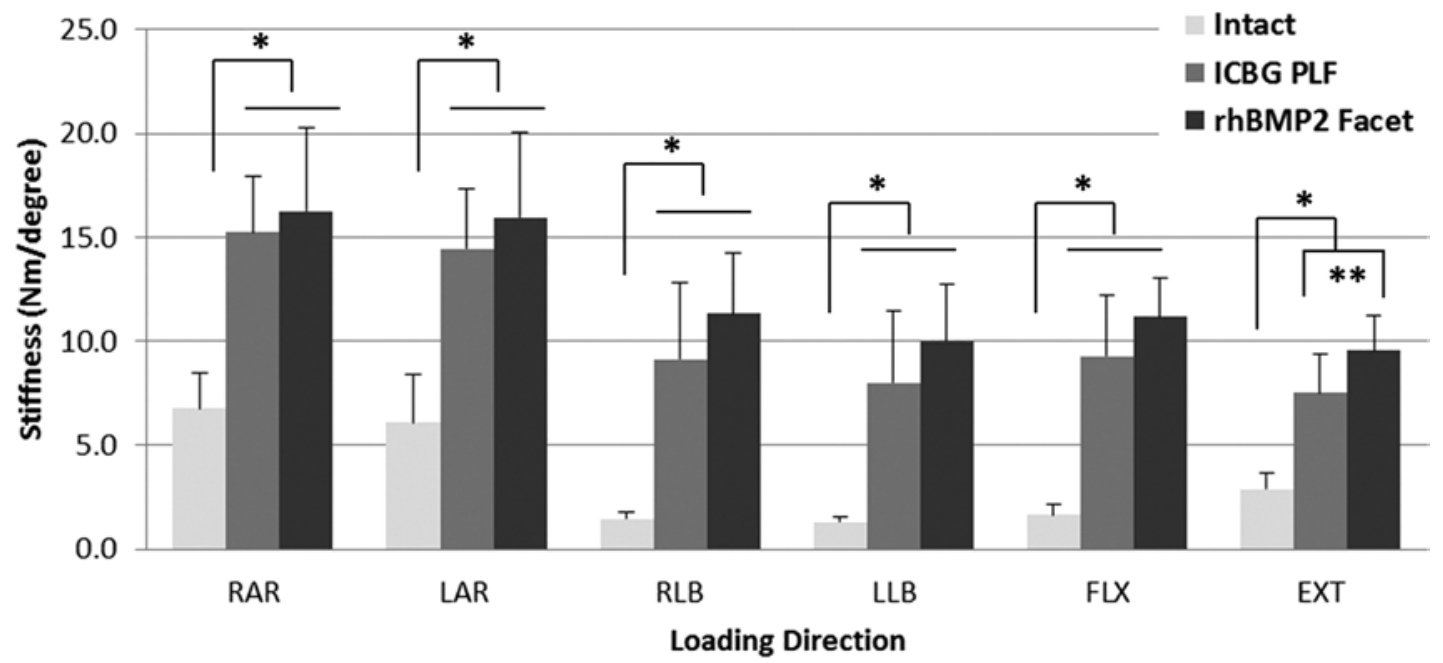

FIG. 3. Mean level stiffness $\left(\mathrm{Nm} /{ }^{\circ}\right)+\mathrm{SD}$ for attempted PLFs with ICBG PLF, as well as attempted facet fusions with rhBMP2 Facet in right axial rotation (RAR), left axial rotation (LAR), right lateral bending (RLB), left lateral bending (LLB), flexion (FLX), and extension (EXT). Historical stiffness data for unoperated normal levels (intact) are also shown for reference purposes. ${ }^{2} \mathrm{p}<0.001$ : ICBG PLF and rhBMP-2 Facet were significantly stiffer than the intact spines in all loading directions. ** $p<0.05$ : rhBMP-2 Facet was significantly stiffer than ICBG PLF in extension. 


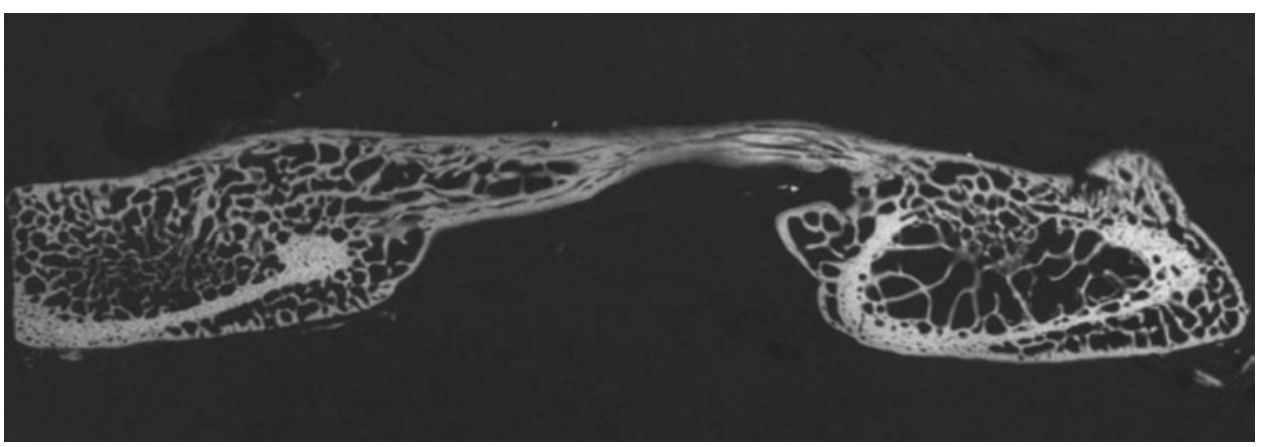

FIG. 4. Microradiograph of a sagittal undecalcified section from the ICBG PLF onlay group demonstrating histological fusion (continuous bony bridging) between the decorticated transverse processes. Original magnification $\times 3$.

Dunn post hoc test). The largest increase in stiffness for the treated segments compared with the intact segments was in the lateral bending direction. The levels of stiffness of the ICBG PLF and rhBMP-2 Facet groups were 6.2 and 7.7 times stiffer, respectively, than intact normal levels in terms of lateral bending. The stiffness increase was 5.6 and 6.7 times the ICBG PLF and rhBMP-2 Facet groups in terms of flexion, respectively. The smallest increase over the normal intact spines was in the axial rotation loading direction at 2.3 and 2.5 times for ICBG PLF and rhBMP2 Facet groups, respectively.

\section{Histological Examination}

Four of the 8 levels in the ICBG PLF treatment group demonstrated bilateral histological fusion, which consisted of continuous bony bridging from superior to inferior on both the right and left sides of the spinal level. A microradiograph of a stained undecalcified sagittal section from the ICBG PLF treatment group showing histological fusion with continuous bony bridging between the transverse processes is presented in Fig. 4. Levels that were determined to be bilaterally fused on histological study had increased values for lateral bending in comparison with those levels that did not have bilateral histological fusion. One of the 8 levels in the ICBG PLF treatment group demonstrated bilateral histological nonfusion with no continuous bony bridging from superior to inferior on the right and left sides of the spinal level. This level had the lowest values for right and left lateral bending in comparison to the other treated levels in this experimental group. In addition, the stiffness of this nonfused level in terms of right and left lateral bending was less than half of the next lowest values for stiffness in terms of right and left lateral bending for this treatment group. Three of the 8 levels in the ICBG PLF treatment group demonstrated unilateral histological fusion that consisted of histological fusion on the left side and histological nonfusion on the right side. The reason for the increased likelihood of left side versus right side fusion is not known.

All 8 levels in the ICBG facet fusion treatment group demonstrated bilateral histological fusion that consisted of continuous bony bridging from superior to inferior on both the right and left sides of the spinal level. A representative, stained, undecalcified sagittal section from the ICBG facet fusion treatment group labeled with anatomical features is shown in Fig. 5A. A representative microra- diograph of a stained, undecalcified sagittal section from the ICBG facet fusion treatment group is shown in Fig. 5B.

All 8 levels in the rhBMP-2 Facet group demonstrated bilateral histological fusion that consisted of continuous bony bridging from superior to inferior on both the right and left sides of the spinal level. A representative microradiograph of a stained, undecalcified sagittal section from the rhBMP-2 Facet group labeled with its anatomical features is shown in Fig. 6A. A representative, stained, undecalcified sagittal section from the rhBMP-2 Facet group is shown in Fig. 6B. The facet fusions in this group were indistinguishable from the facet fusions with autograft in terms of bone quality, size and shape of the fusion mass, and bone remodeling. No evidence of bone encroachment into the neuroforaminal space was evident in the undecalcified histological specimens and corresponding microradiographs of the facetectomy defects treated with rhBMP-2 or ICBG. Six of 16 treated facet defects in the rhBMP-2 Facet group demonstrated small blood-filled cysts or seromas that resulted in small radiolucent voids within the treatment areas in the corresponding microradiographs. This phenomenon was not observed in the other treatment groups. A stained undecalcified sagittal section from the rhBMP-2 Facet group showing a hematoma within a fluidfilled void within the fusion mass is seen in Fig. 6C. Intraosseous seromas/hematomas had no apparent impact on fusion stiffness.

\section{Discussion}

The present study compared the efficacies of 3 different lumbar fusion techniques in an instrumented ovine model. When used for facet fusions in the instrumented ovine lumbar facetectomy model, both ICBG and rhBMP-2/ ACS demonstrated efficacy by generating a $100 \%$ histological fusion rate. Only 4 of the 8 levels in the ICBG PLF treatment group demonstrated bilateral histological fusion. One of the 8 levels in the ICBG PLF treatment group demonstrated bilateral histological nonfusion. Three of the 8 levels in the ICBG PLF treatment group demonstrated unilateral histological fusion, with histological fusion occurring on the left side and histological nonfusion occurring on the right side. When used for lumbar PLF in humans, Boden summarized the literature to indicate that autograft is associated with a nonunion rate that ranges from $10 \%$ to $40 \%$ with single-level fusions. ${ }^{1}$ A higher non- 


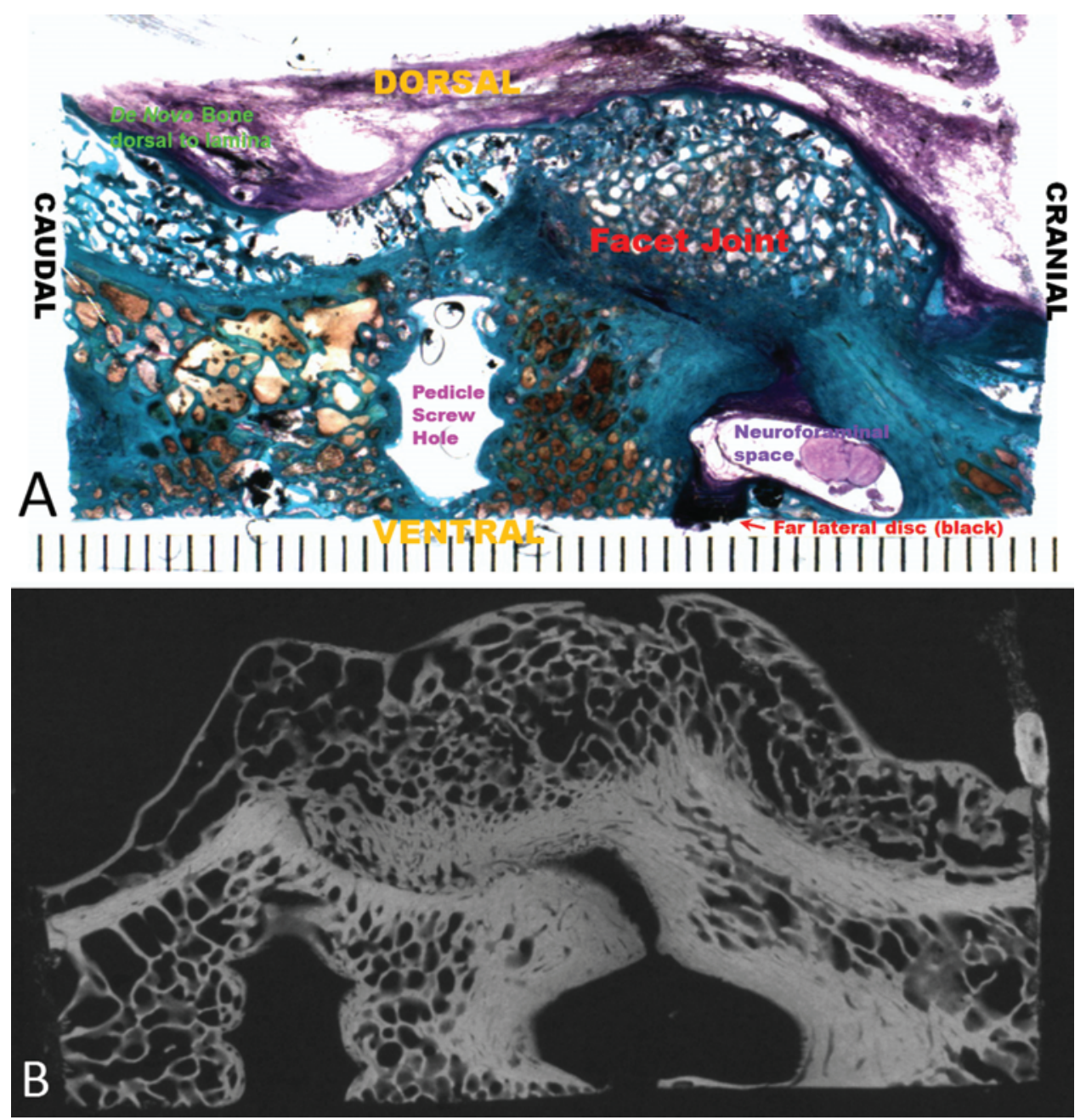

FIG. 5. A: A trichrome-stained, undecalcified sagittal section from the autograft facet fusion treatment group demonstrating histological fusion (continuous bony bridging) through the previous facet joint as well as dorsal to the facet joint. A millimeter scale is shown at the bottom of the image. B: Microradiograph of a stained, undecalcified sagittal section from the autograft facet fusion treatment group demonstrating histological fusion (continuous bony bridging) through the previous facet joint as well as dorsal to the facet joint. Original magnification $\times 3$. Figure is available in color online only.

union rate is associated with attempted fusions at multiple levels. ${ }^{1}$ In a randomized prospective study, France et al. found no statistically significant differences in the fusion rate when pedicle screw instrumentation was used with an autograft in comparison with uninstrumented fusion with an autograft. ${ }^{4}$ In the ovine PLF model, the arthrodesis rate varies between $25 \%$ and $100 \%$ at 4 to 24 months when spinal segments are evaluated with manual palpation, radiography, and/or histology. ${ }^{6-9,16}$ However, similar to the clinical situation, fusion rates can be lower in the ovine model when multiple levels are fused..$^{9}$ Thus, based on the data from the current study as well as the historical literature, the ovine lumbar PLF model appears to be a valid model not only biomechanically, but also with respect to fusion outcomes, which are similar to those found clinically.

In the current study, radiography and undecalcified histology showed that when the ICBG material was used for the attempted PLF, fusion occurred, although in most of the cases the location of the fusion was not found to be in the middle of the intertransverse process space (i.e., be- tween transverse processes) in the majority of the L2-3 levels. We suspect that the graft material may have been placed or displaced medially under the rod so that for attempted the PLFs with ICBG at L2-3, fusion was found more medially in the spines and dorsal to the facet joints as well as dorsal to the laminae. Thus, similar to the fusion masses with attempted facet fusions, undecalcified histology and the corresponding microradiography demonstrated more medial fusions dorsal to the facet joints in the autograft posterolateral onlay fusion group despite the different surgical techniques used.

The biomechanics data demonstrated that PLF with ICBG and facet fusion with rhBMP-2/ACS were significantly stiffer than the intact controls in all 6 loading directions. Biomechanically, the ICBG PLF and the rhBMP-2 Facet groups demonstrated similar levels of stiffness in all 6 loading directions, with the rhBMP-2 Facet group having slightly higher average stiffness in all directions. However, it should be noted that the ICBG PLF group was heterogeneous with respect to histological fusion. Thus, 


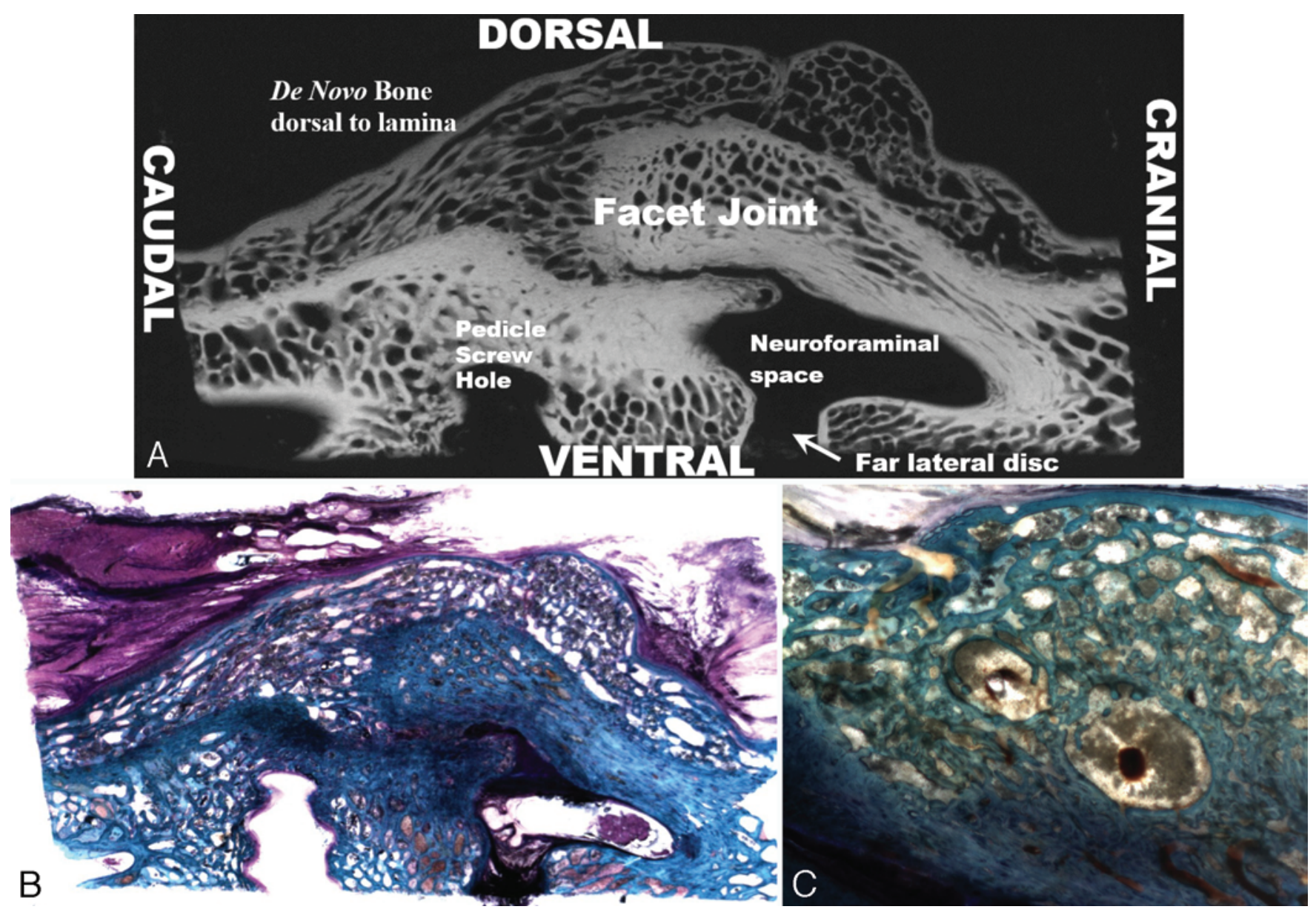

FIG. 6. A: Microradiograph of an undecalcified sagittal section from the rhBMP-2 Facet group demonstrating histological fusion (continuous bony bridging) through the previous facet joint as well as dorsal to the facet joint. Original magnification $\times 3$. B: A trichrome-stained, undecalcified sagittal section from the rhBMP-2 Facet group demonstrating histological fusion (continuous bony bridging) through the previous facet joint as well as dorsal to the facet joint. Original magnification $\times 3$. C: Trichrome-stained, undecalcified sagittal histology from the rhBMP-2/ACS Facet group showing a hematoma within a fluid-filled void within the fusion mass. Original magnification $\times 80$. Figure is available in color online only.

if a comparison of the biomechanical data from just the fused PLFs was made to the biomechanical data from the rhBMP-2 Facet group, the results might have been different. The similarities in the biomechanical results might be explained by the fact that the undecalcified histology and corresponding microradiography demonstrated similar anatomical locations of fusions despite the different surgical techniques used. Thus, during mechanical testing, comparable fusion masses resisted mechanical loading in a similar fashion.

One limitation of this study was the lack of a sham control, which might consist of a facetectomy defect without the application of a bone graft or bone graft substitute. Such an experimental control could establish if spontaneous facet fusion can occur in the instrumented ovine model. In addition, because 3 experimental fusion treatments were attempted in 3 contiguous levels in this model, the success of PLF with ICBG may have been lower than if a single-level PLF was attempted in each sheep. A potential limitation of the current study is that we did not randomize the treatments to different lumbar levels in the sheep. We do not think this is a major limitation of the study as Wilke et al. showed that the stiffness parameters, neutral zone, and range of motion for sheep lumbar levels from L2-3 to L4-5 were equivalent and showed very little variation between these lumbar segments. ${ }^{18}$

One unusual finding in this study was the presence of both fluid-filled cysts and blood-filled cysts. Six of 16 treated facet defects in the rhBMP-2 Facet group demonstrated small blood-filled cysts or seromas in the bony treatment area that resulted in radiolucent voids in the corresponding microradiographs. The cause of these radiolucent areas adjacent to the treatment is not known. Tannoury and An provide a review of the literature with respect to the complications of rhBMP-2/ACS in spine surgery..$^{15}$ While the authors summarize several reports on the clinical incidence of both seromas and hematomas associated with rhBMP-2 usage, no cause for the hematomas or seromas has been reported in the literature..$^{15}$

While 6 months provides valuable data on the efficacy of these treatments to induce spine fusion in a validated animal model, more studies and complementary data are needed to further assess these treatments for clinical use. For example, we used supplemental instrumentation be- 
cause this is consistent with current clinical practice for PLFs. ${ }^{14}$ However, an uninstrumented ovine model might be investigated to determine if this affects the facet fusion rate with ICBG and rhBMP-2/ACS. For example, clinically, Pateder and Benzel found that uninstrumented facet fusion decreases motion and stabilizes the spine in a retrospective analysis of uninstrumented facet fusions in patients undergoing lumbar laminectomy for spinal stenosis due to single-level degenerative spondylolisthesis. ${ }^{11}$ Also, although the biomechanical stiffness of the fusion segments was assessed in a nondestructive manner in the current study, a destructive test measuring the load to failure was not performed. Only the stiffness of the fusion segment was assessed in the current study with the use of nondestructive biomechanics. Future complementary studies might look at the maximum load-carrying capability of the fusion constructs using destructive testing.

\section{Conclusions}

Both rhBMP-2/ACS and ICBG demonstrated 100\% efficacy when used for facet fusion in the instrumented ovine model. However, the ICBG PLF treatment group only demonstrated a $50 \%$ bilateral histologic fusion rate. Biomechanically, the ICBG PLF and rhBMP-2 Facet groups demonstrated similar levels of stiffness in all 6 loading directions, with the rhBMP-2 Facet group having on average slightly higher stiffness in all directions.

\section{Acknowledgments}

The authors thank and acknowledge funding from Medtronic Spinal and Biologics for providing research support for this study. The authors wish to thank and acknowledge Henry Kuhnen and Mike Siebert for their technical expertise in producing undecalcified histological sections as well as Linda McGrady for technical expertise in biomechanical testing.

\section{References}

1. Boden SD: Overview of the biology of lumbar spine fusion and principles for selecting a bone graft substitute. Spine (Phila Pa 1976) 27 (16 Suppl 1):S26-S31, 2002

2. Easley NE, Wang M, McGrady LM, Toth JM: Biomechanical and radiographic evaluation of an ovine model for the human lumbar spine. Proc Inst Mech Eng H 222:915-922, 2008

3. Foley KT, Holly LT, Schwender JD: Minimally invasive lumbar fusion. Spine (Phila Pa 1976) 28 (15 Suppl):S26-S35, 2003

4. France JC, Yaszemski MJ, Lauerman WC, Cain JE, Glover $\mathrm{JM}$, Lawson KJ, et al: A randomized prospective study of posterolateral lumbar fusion. Outcomes with and without pedicle screw instrumentation. Spine (Phila Pa 1976) 24:553-560, 1999

5. Geiger M, Li RH, Friess W: Collagen sponges for bone regeneration with rhBMP-2. Adv Drug Deliv Rev 55:16131629,2003

6. Guigui P, Plais PY, Flautre B, Viguier E, Blary MC, Sales De Gauzy J, et al: Experimental model of posterolateral spinal arthrodesis in sheep. Part 1. Experimental procedures and results with autologous bone graft. Spine (Phila Pa 1976) 19:2791-2797, 1994

7. Gupta MC, Theerajunyaporn T, Maitra S, Schmidt MB, Holy CE, Kadiyala $S$, et al: Efficacy of mesenchymal stem cell enriched grafts in an ovine posterolateral lumbar spine model. Spine (Phila Pa 1976) 32:720-727, 2007
8. Jahng TA, Fu TS, Cunningham BW, Dmitriev AE, Kim DH: Endoscopic instrumented posterolateral lumbar fusion with Healos and recombinant human growth/differentiation factor-5. Neurosurgery 54:171-181, 2004

9. Mermer MJ, Gupta MC, Wheeler DL, Helgerson J, Reddi AH, Hazelwood S, et al: Efficacy of osteogenic protein-1 in a challenging multilevel fusion model. Spine (Phila Pa 1976) 29:249-256, 2004

10. Park YK, Kim JH, Oh JI, Kwon TH, Chung HS, Lee KC: Facet fusion in the lumbosacral spine: a 2-year follow-up study. Neurosurgery 51:88-96, 2002

11. Pateder DB, Benzel E: Noninstrumented facet fusion in patients undergoing lumbar laminectomy for degenerative spondylolisthesis. J Surg Orthop Adv 19:153-158, 2010

12. Sandhu HS, Kanim LEA, Girardi F, Cammisa FP Jr, Dawson ED: Animal models of spinal instability and spinal fusion, in An YH, Friedman RJ (eds): Animal Models in Orthopaedic Research. Boca Raton, FL: CRC Press, 1999, pp 505-526

13. Smit TH: The use of a quadruped as an in vivo model for the study of the spine-biomechanical considerations. Eur Spine J 11:137-144, 2002

14. Steinmann JC, Herkowitz HN: Pseudarthrosis of the spine. Clin Orthop Relat Res (284):80-90, 1992

15. Tannoury CA, An HS: Complications with the use of bone morphogenetic protein 2 (BMP-2) in spine surgery. Spine J 14:552-559, 2014

16. Wheeler DL, Jenis LG, Kovach ME, Marini J, Turner AS: Efficacy of silicated calcium phosphate graft in posterolateral lumbar fusion in sheep. Spine J 7:308-317, 2007

17. White AA, Panjabi MM (eds): Clinical Biomechanics of the Spine, ed 2. Philadelphia: Lippincott Williams \& Wilkins, 1990

18. Wilke HJ, Kettler A, Claes LE: Are sheep spines a valid biomechanical model for human spines? Spine (Phila Pa 1976) 22:2365-2374, 1997

\section{Disclosures}

Both The Medical College of Wisconsin and Colorado State University received research funding paid directly to their institutions from Medtronic Spinal and Biologics in support of the study. Dr. Foley received clinical or research support for the study described from Medtronic, serves as a scientific consultant for Medtronic, holds patents with Medtronic and NuVasive, receives royalties for patents from Medtronic, and owns shares in Derma Sciences, Discgenics, Medtronic, NuVasive, SpineWave, and TrueVision. Dr. Toth received clinical or research support for the study described from Medtronic, serves as a scientific consultant for Medtronic, and has served as a scientific expert for Cytophil Inc.

\section{Supplemental Information \\ Previous Presentations}

Portions of this work were presented in abstract form at the 26th Annual Meeting of the North American Spine Society, Chicago, Illinois, November 1-5, 2011.

\section{Author Contributions}

Conception and design: Toth, Foley. Acquisition of data: Toth, Wang, Seim, Turner. Analysis and interpretation of data: Toth, Foley, Wang. Drafting the article: Toth, Foley. Critically revising the article: Toth, Foley, Wang. Reviewed submitted version of manuscript: Toth. Approved the final version of the manuscript on behalf of all authors: Toth. Study supervision: Toth.

\section{Correspondence}

Jeffrey M. Toth, Department of Orthopaedic Surgery, The Medical College of Wisconsin Inc., 9200 W Wisconsin Ave., Box 26099, Milwaukee, WI 53226-0099. email: jtoth@mcw.edu. 